\title{
Polyether ether ketone implants achieve increased bone fusion when coated with nano-sized hydroxyapatite: a histomorphometric study in rabbit bone
}

\author{
This article was published in the following Dove Press journal: \\ International Journal of Nanomedicine \\ 6 April 2016 \\ Number of times this article has been viewed
}

\section{Pär Johansson' \\ Ryo Jimbo' \\ Yoshihito Naito ${ }^{2}$ \\ Per Kjellin ${ }^{3}$ \\ Fredrik Currie ${ }^{3}$ \\ Ann Wennerberg'}

'Department of Prosthodontics, Faculty of Odontology, Malmö University, Malmö, Sweden; ${ }^{2}$ Oral Implant Center, Tokushima University Hospital, Tokushima, Japan; ${ }^{3}$ Promimic AB, Stena Center, Göteborg, Sweden
Correspondence: Pär Johansson Department of Prosthodontics, Faculty of Odontology, Malmö University, Carl Gustafs väg 34, 21421 Malmö, Sweden Tel +464066585I4 Email par.johansson@mah.se

\begin{abstract}
Polyether ether ketone (PEEK) possesses excellent mechanical properties similar to those of human bone and is considered the best alternative material other than titanium for orthopedic spine and trauma implants. However, the deficient osteogenic properties and the bioinertness of PEEK limit its fields of application. The aim of this study was to limit these drawbacks by coating the surface of PEEK with nano-scaled hydroxyapatite (HA) minerals. In the study, the biological response to PEEK, with and without HA coating, was investigated. Twenty-four screw-like and apically perforated implants in the rabbit femur were histologically evaluated at 3 weeks and 12 weeks after surgery. Twelve of the 24 implants were HA coated (test), and the remaining 12 served as uncoated PEEK controls. At 3 weeks and 12 weeks, the mean bone-implant contact was higher for test compared to control $(P<0.05)$. The bone area inside the threads was comparable in the two groups, but the perforating hole showed more bone area for the HA-coated implants at both healing points $(P<0.01)$. With these results, we conclude that nano-sized HA coating on PEEK implants significantly improved the osteogenic properties, and in a clinical situation this material composition may serve as an implant where a rapid bone fusion is essential.
\end{abstract}

Keywords: HA, PEEK, osseointegration, histology, orthopedics, in vivo

\section{Introduction}

Few new materials are introduced to the medical device industry, partly because of the good quality of the existing ones and the extremely high cost for introducing new ones. Despite this, polyether ether ketone (PEEK) was introduced where metallic implants such as $\mathrm{Ti}$ alloys and $\mathrm{CoCr}$ alloys experienced inferior outcomes due to their mechanical properties. ${ }^{1-5}$ One such potential shortcoming of conventional implant materials is the tenfold higher elastic modulus of titanium compared to bone that has been found to cause degeneration of adjacent segments via stress shielding. To overcome these problems, PEEK was introduced into the orthopedic field as an implant material due to its similar elastic modulus as that of human cortical bone. ${ }^{6}$ For the past 2 decades, PEEK has become a common alternative to metal implants in several orthopedic applications. ${ }^{7,8}$

However, PEEK has shown both in vivo and in vitro studies to have low bioactive features due to the relatively bioinert surface. ${ }^{9-11}$ Therefore, many studies have been conducted to improve the biocompatible state of PEEK by incorporating bioactive substances into the substrate or onto the surface as a coating. ${ }^{12-14}$ The authors have 
found that incorporation of hydroxyapatite (HA) into the PEEK compound substantially decreased the mechanical properties of the material. ${ }^{14,15}$ Instead, by altering the surface topography and chemical composition, the surface becomes more bioactive and an improved bone formation can be achieved owing to an increased surface area and higher surface energy. ${ }^{3}$ Therefore, several techniques to coat the PEEK surface have been evaluated. Plasma sprayed deposition and electron beam deposition are two common methods to coat the surface with HA, which improved the bioactive potential of PEEK. However, the long-term effect of these techniques on titanium has been questioned after retrieved sections have demonstrated inflammation cells adjacent to detached coating fragments. The coating thickness and mechanical attachment to the substrate were hypothesized to be one reason to cause this incident. ${ }^{16-18}$

In this study, a liquid-based nano-sized HA solution was applied to a PEEK surface with a wet chemically based technique, and the excess was removed with pressurized gas and by spinning the implant. The final step in this coating method is a brief heat treatment, which is performed at a temperature just below the melting point of the PEEK polymer. This will create a sintering effect that adheres the crystals to the PEEK substrate. The end result is an extremely thin layer of ceramic coating where the risk of delamination becomes minimal. In other studies, this exceptional technique has been found to successfully increase the bone formation on titanium but it has never been applied on PEEK. ${ }^{9-21}$ Using HA in the form of nanoparticles increases not only the surface area but also the interaction and adhesion between the crystals and the PEEK surface. Another unique feature of this study was a perforating hole of the implant that permits evaluation of the osteoconductive effect of the surface modification. Osteoconductive ability is a decisive feature when bone fusion is requested, for instance, in several spinal applications.

The aim of this study was to evaluate the bone formation around and bone conductivity into the apical hole of the PEEK implant (Figure 1). One group was coated with nanocrystalline HA, and the comparison was made against an untreated PEEK implant. The experiment was carried out in a well-documented rabbit femur model.

\section{Materials and methods Implant surface preparation}

Forty-eight noncutting PEEK implants (Invibio Ltd, Lancashire, UK) with a diameter of $3.5 \mathrm{~mm}$ and a length of $4 \mathrm{~mm}$ were prepared for the study. The implants were machined with a hole penetrating the apical parts to provide

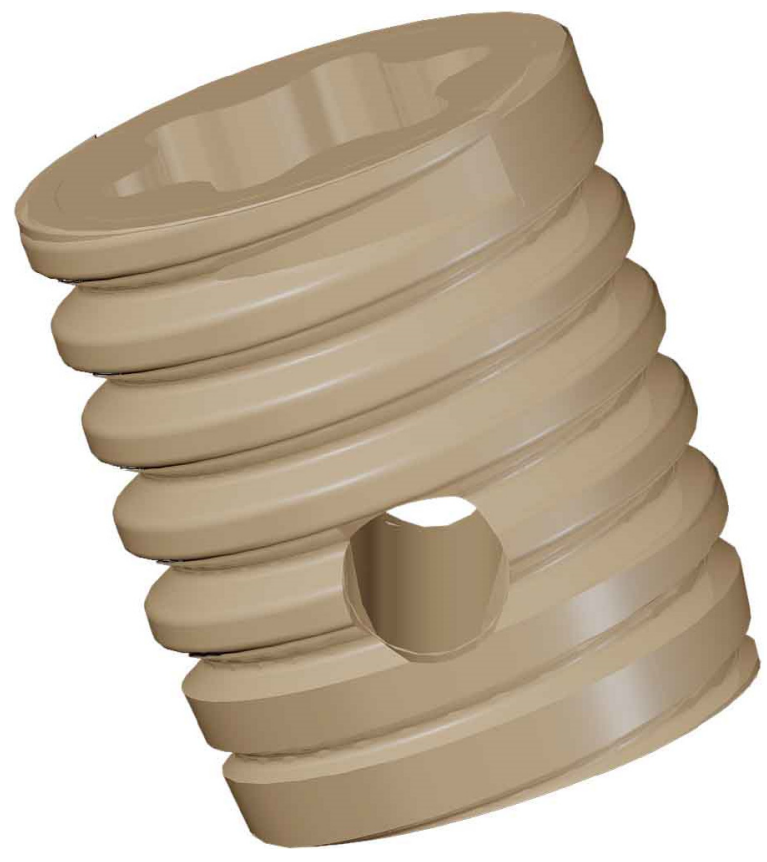

Figure I A 3D rendered image of the PEEK implant.

Abbreviations: 3D, three-dimensional; PEEK, polyether ether ketone.

a fully intact coated surface, even after insertion, which allow evaluation of the bone ingrowth (Figure 1). This unique design permits evaluation of the surface osteoconductivity where the amount of newly formed bone within the hole can be measured. The surface of half the total amount of implants $(n=24)$ was coated with the $\mathrm{HA}^{\text {nano }}$ Surface provided by Promimic AB, Göteborg, Sweden (test), whereas the remaining implants $(n=24)$ were used with their native surfaces (control). For the implants to be coated, $50 \mu \mathrm{L}$ of coating solution was applied on top of each implant. The implant was then rotated at 2,700 rpm for 5 seconds, in order to create a homogenous layer of coating liquid onto the implant surface. The coating solution was a microemulsion, containing nano-sized HA crystals, $20-50 \mathrm{~nm}$ long and 2-10 nm wide, dispersed with water, surfactants, and organic solvent. The coating solution is more thoroughly described in a patent description..$^{22}$ After the coating procedure, the PEEK implant was put into an oven having an oxygen-enriched atmosphere at $325^{\circ} \mathrm{C}$ for 5 minutes. This heat treatment was done to remove the surfactants and to adhere the crystals onto the PEEK surface. The thickness of the resulting HA layer was $20-40 \mathrm{~nm}$.

\section{Morphological characterization}

The surface morphology of the study's assigned PEEK implants was performed with a scanning electron microscopy (LEO Ultra 55 FEG; ZEISS, Oberkochen, Germany) 
at an accelerating voltage of $2-4 \mathrm{kV}$. To make the surface conductive, the implants were sputtered with gold, using a JEOL JFC-1100E (JEOL Ltd, Tokyo, Japan) operating at $10 \mathrm{kV}$ for 90 seconds. The nano and micro topographies of the implants are described in a previous report. ${ }^{23}$

\section{Surgical procedure and implantation}

The animal study was approved by the Malmö/Lund (Sweden) regional animal ethics committee and all experiments were performed following the institutional and national guidelines and regulations of the Board of Agriculture. Twenty-four Swedish lop-eared rabbits (mean weight $4.1 \mathrm{~kg}$ ) received one HA and one control implant each, which were randomly inserted into the cancellous bone of the left and right distal femur. The rabbits were divided into two groups $(n=12)$ for observation periods of either 3 weeks or 12 weeks after implant surgery. The healing times, 3 weeks and 12 weeks, refer to the early stage of bone formation and a complete bone healing, respectively. An anesthetic dose of $0.15 \mathrm{~mL} / \mathrm{kg}$ medetomidine (1 mg/mL Domitor; Orion Pharma, Sollentuna, Sweden) and $0.35 \mathrm{~mL} / \mathrm{kg}$ ketamine hydrochloride (50 mg/mL Ketalar; Pfizer Inc., New York, NY, USA) was administered. The implant site was disinfected (70\% ethanol; Solveco AB, Rosersberg, Sweden, and $5 \mathrm{mg} / \mathrm{mL}$ chlorhexidine; Fresenius Kabi AB, Uppsala, Sweden), and additional local anesthesia (lidocaine hydrochloride, Xylocaine; AstraZeneca plc, Södertälje, Sweden) was injected subcutaneously before a skin incision was made to expose the bone. The bone was trephined with a series of drills up to a final diameter of $3.2 \mathrm{~mm}$. Tapping and insertion was performed manually before the implant was installed within the femoral condyle (Figure 2A and B). This implant location provides bone with trabecular structure that covers the entire implant surface. The wound was cleaned and generously irrigated with sterile saline before the fascia and skin was closed separately with bioresorbable sutures (Ethicon, Norderstedt, Germany). Postsurgical analgesic buprenorphine hydrochloride $(0.5 \mathrm{~mL}$ Temgesic; Reckitt Benckiser, Slough, UK) was administrated the next 3 days. The animals were euthanized with an overdose of sodium pentobarbital $(60 \mathrm{mg} / \mathrm{mL}$; Apoteksbolaget AB, Stockholm, Sweden). Bone-implant blocks (Figure 2A and $B$ ) were retrieved en bloc, immersed, and fixated in $4 \%$ buffered formaldehyde for 24 hours.

\section{Histomorphometry}

The bone-implant blocks were increasingly dehydrated in graded ethanol up to $99.9 \%$ (Solveco AB). Gradually, the ethanol was cleared and the tissue was simultaneously infiltrated with resin without decalcification. Finally, the specimens were embedded in light-curing resin (Technovit 7200 VLC; Heraeus Kulzer, Wehrheim, Germany).

The samples were cut and ground using a diamond blade and grinding system (Exakt; Apparatebau, Norderstedt, Germany) into a final thickness of $\sim 20 \mu \mathrm{m}$ and thereafter stained with toluidine blue. The cut was aligned along the apical hole in order to evaluate bone area (BA), bone-implant contact (BIC), and BA inside the hole on the same histological section. The evaluation was carried out under a light microscopy (Eclipse ME600, Nikon Corporation, Tokyo, Japan). The percentage of BIC and new BA in the threads
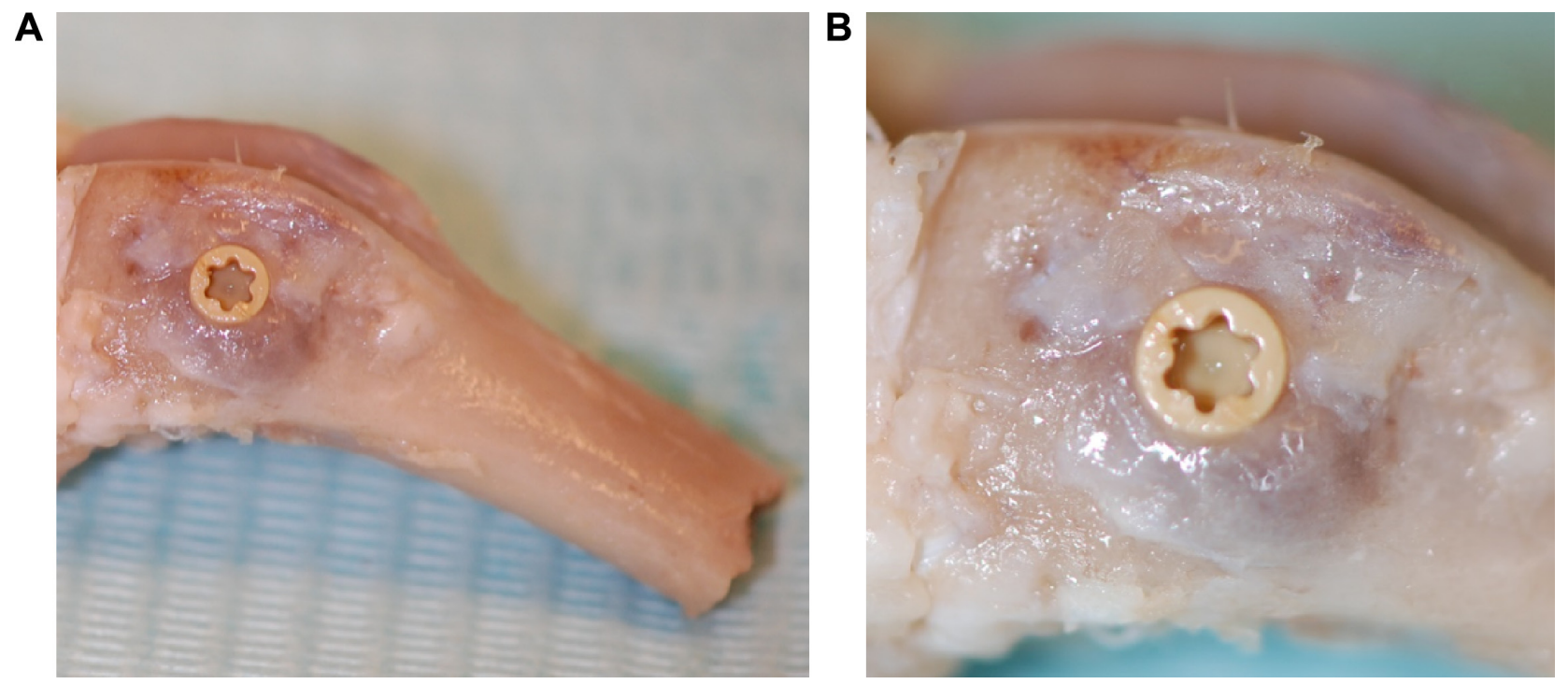

Figure 2 Clinical images of bone block (A) after retrieval demonstrating implant positioning and (B) with a magnified image of the implant in situ. 


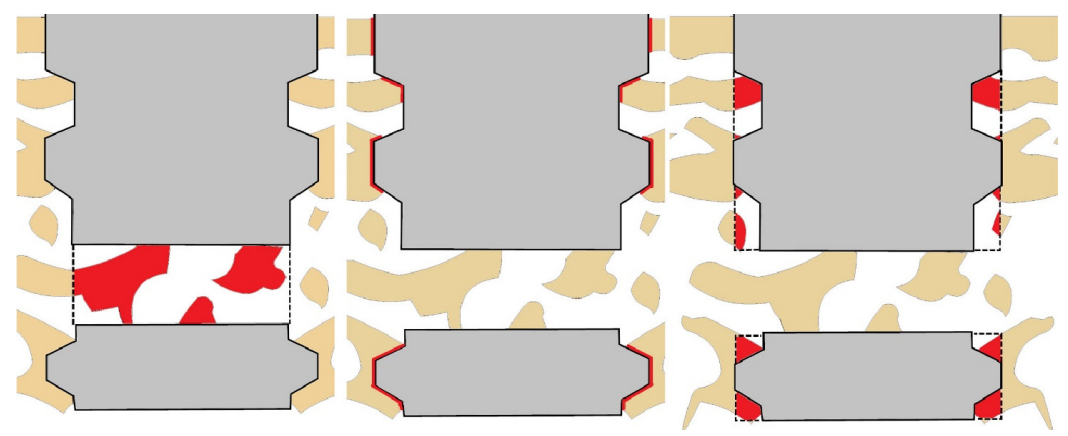

Figure 3 A schematic image of the area of interest in the quantitative histomorphometric analysis.

Notes: The measured area of interest is colored red and bone is colored tan. Bone area inside the perforating hole (left), bone-implant contact (middle), and bone area inside threads (right).

and inside the hole were calculated with image analysis software (ImageJ Version 1.43r; National Institutes of Health, Bethesda, MD, USA). Figure 3 shows a schematic illustration of the histomorphometric measurement areas.

\section{Statistics}

The histological data were analyzed with a nonparametric Mann-Whitney test with a level of significance set at 0.05. The analysis was performed using SPSS software (Version 20; IBM Corporation, Armonk, NY, USA).

\section{Results}

\section{Morphological and topographical analyses}

Scanning electron micrograph images of the two types of implant surfaces and their typical surface features are given in Figure 4A and B, for two magnifications. At a higher magnification $(80 \mathrm{~K})$ of the coated surface, the HA minerals appear as rod-shaped particles fully covering the PEEK surface (Figure 4B). The rods are arranged in different directions with a size of $\sim 10 \mathrm{~nm}$ width and $150 \mathrm{~nm}$ length. At a lower resolution $(40 \mathrm{~K})$, there were no remarkable topographical differences between the two surfaces, with the possible exception that the HA particles have leveled out the striations from the turning procedure (Figure 4A).

\section{Histomorphometry}

At the time of implant retrieval, there were no clinical signs of infection and all the implants were immobilized without any visible signs of marginal bone resorption. The qualitative histological evaluation revealed stained woven bone close to the implant surface and deeper stained lamellar bone in proximity to the implants (Figure 5A and D). A Haversian system can be seen within the thread of an HA-coated surface after 12 weeks of healing (Figure 5C). No immunological cells were seen in proximity to the implanted materials. In the perforated hole at 3 weeks, the bone had extended $>50 \%$ of the diameter of the hole with irregular contact to the inner wall of the implant (Figure 5A and B).

The quantitative evaluation of the BIC revealed newly formed bone in contact with the implant surface. The BIC after 3 weeks was significantly higher for the test implants with a value of $14.1 \% \pm 3.5 \%$ compared to $11.1 \% \pm 3.5 \%$ for
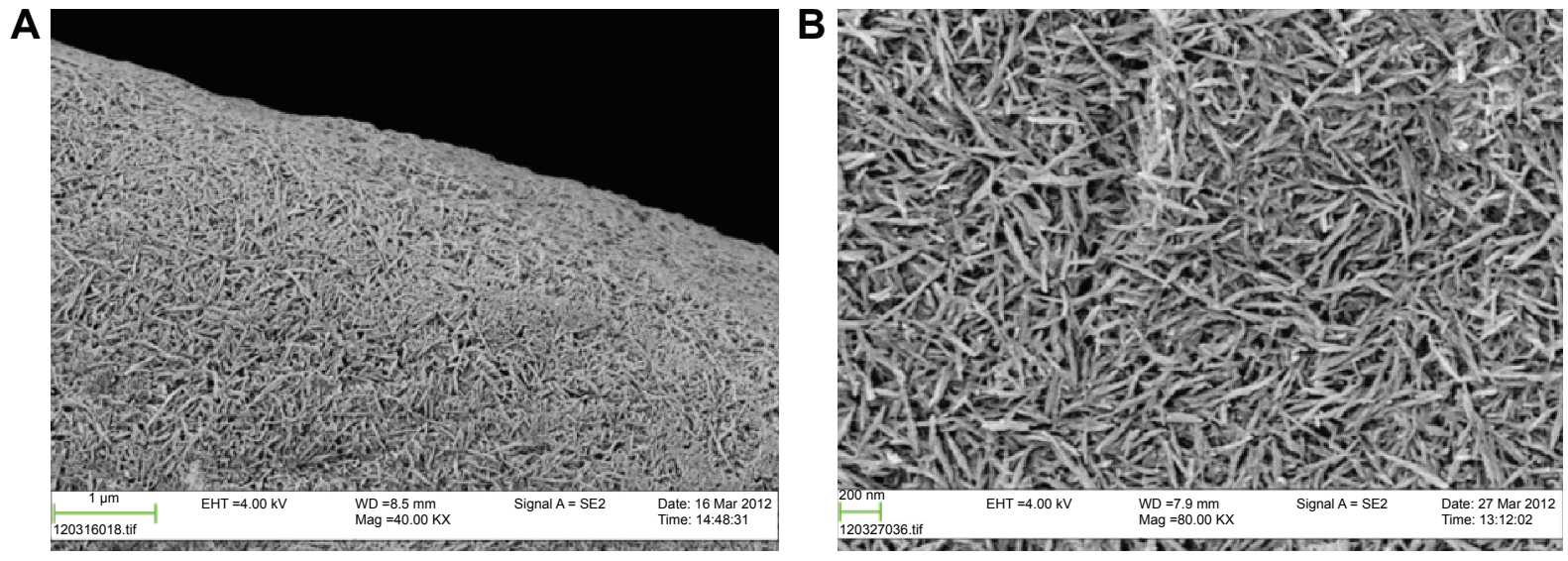

Figure 4 SEM photographs of hydroxyapatite-coated PEEK in two magnifications: 40K (A) and 80K (B). Abbreviations: SEM, scanning electron microscopy; PEEK, polyether ether ketone. 

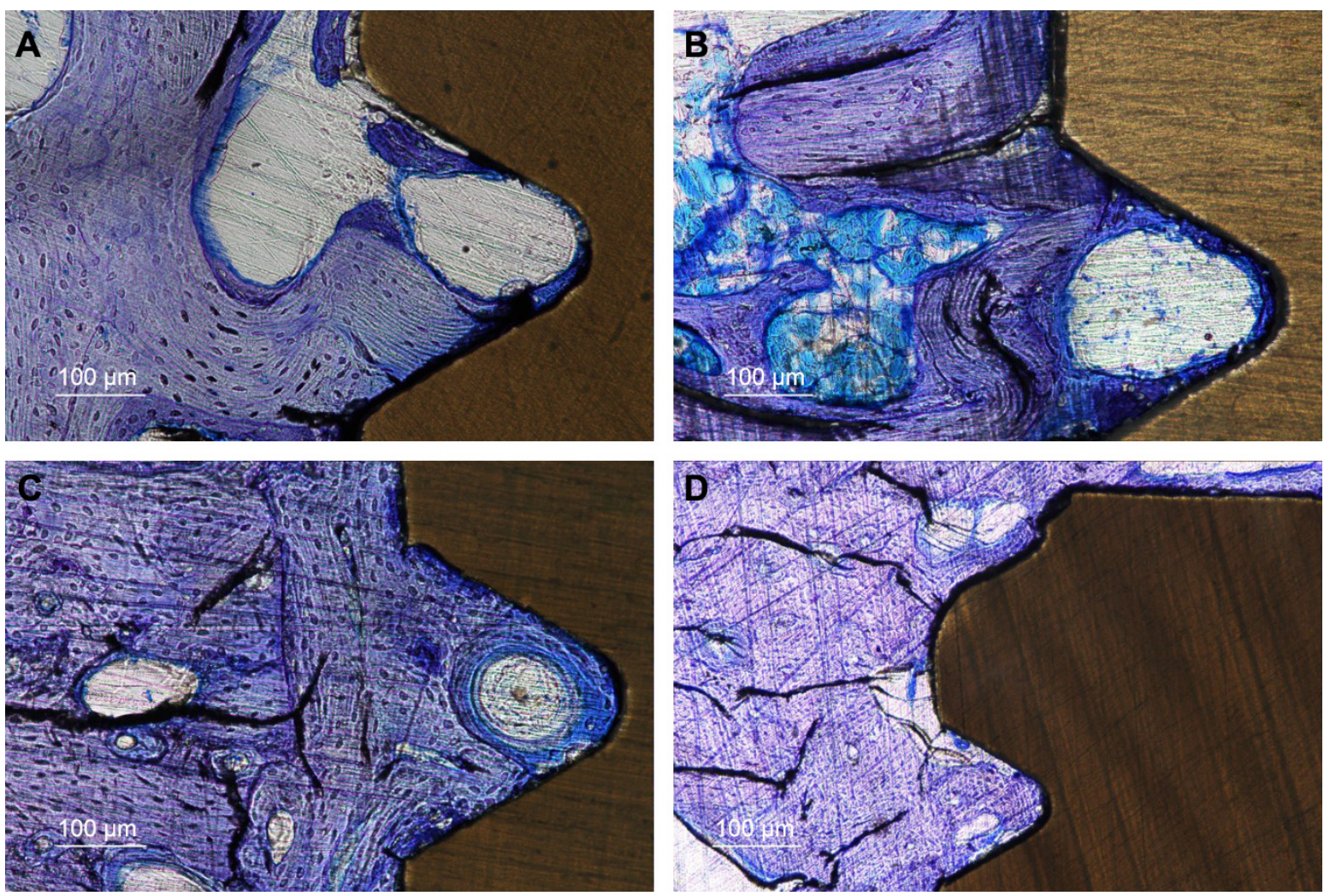

Figure 5 Ground sections $\times 10$ of HA-coated and pure PEEK implants.

Notes: Three weeks after insertion, the HA-coated PEEK (A) shows more osteocytes and lamellar bone compared to the pure PEEK (B). After 12 weeks of healing, the HA-coated PEEK (C) shows signs of vital and rapid bone formation with a Haversian system inside the thread surrounded by large amounts of osteocytes. The uncoated PEEK implant after 12 weeks also demonstrates large amounts of osteocytes but with a lower extent of bone-implant contact (D).

Abbreviations: HA, hydroxyapatite; PEEK, polyether ether ketone.

the control. After 12 weeks, the BIC was also found to be significantly higher for the test group $(P=0.038$; Table 1 and Figure $6 \mathrm{~A}-\mathrm{C})$. Comparing the $\mathrm{BA}$ in the threads of the two groups revealed no significant difference at any healing time point (3 weeks: $P=0.478,12$ weeks: $P=0.211$ ). However, the BA in the apical hole demonstrated a significant difference for the test group ( 3 weeks: $P=0.00,12$ weeks: $P=0.01$ ). At 3 weeks healing, the mean BA in the hole for the test and control was $17.2 \% \pm 7.8 \%$ and $4.52 \% \pm 2.9 \%$, respectively. The BA after 12 weeks demonstrated a significant difference

Table I Summary of the histomorphometric measurements

\begin{tabular}{|c|c|c|c|}
\hline & BIC (\%, SD) & BA-thread $(\%$, SD) & BA-hole (\%, SD) \\
\hline \multicolumn{4}{|l|}{3 weeks } \\
\hline Control & II.08 (3.49) & $25.04(7.64)$ & $4.52(2.9)$ \\
\hline Test & I4.07 (3.54) & $27.68(8.3 I)$ & $|7.2|(7.75)$ \\
\hline$P$-value & 0.034 & 0.478 & 0.000 \\
\hline \multicolumn{4}{|l|}{12 weeks } \\
\hline Control & II.39 (3.8I) & $44.48(10.78)$ & $10.80(5.53)$ \\
\hline Test & $16.65(6.74)$ & $49.66(13.56)$ & $21.33(6.94)$ \\
\hline$P$-value & 0.038 & 0.211 & 0.001 \\
\hline
\end{tabular}

Note: Bold $P$-values show significant values.

Abbreviations: BIC, bone-implant contact; BA, bone area; SD, standard deviation. with mean values for test implants of $21.3 \% \pm 6.9 \%$ and for control implants of $10.8 \% \pm 5.5 \%$. Figure $7 \mathrm{~A}$ shows a coated PEEK implant after 3 weeks of healing where the bone has managed to migrate through the entire hole. In Figure 7B, the line between old and new bones is clear to distinguish and the bone extends though the hole while making contact with the implant surface at regular intervals.

Figure 7A shows a coated PEEK implant after 3 weeks of healing where the bone has managed to migrate through the entire hole. In Figure 7B, the line between old and new bones is clear to distinguish and the bone extends through the hole while making contact with the implant surface at regular intervals.

\section{Discussion}

The aim of this experimental study was to evaluate the effect of a nano-sized HA coating on bone formation of PEEK implants after 3 and 12 weeks of healing in a rabbit femur model. We found that osseointegration of HA-coated PEEK was higher compared with uncoated PEEK implants. HA coating on PEEK significantly improved the BIC at both 

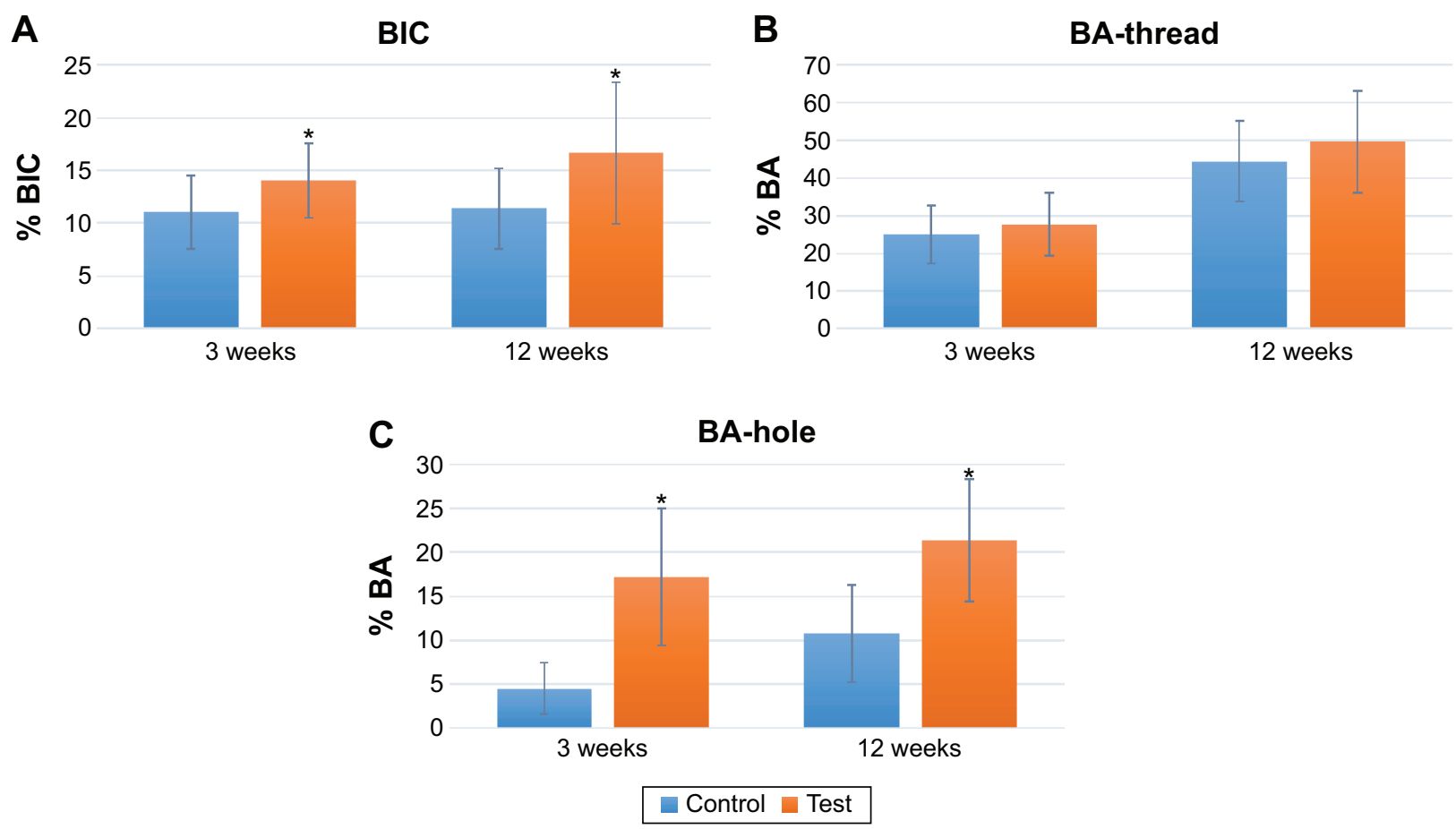

Figure 6 Graphs presenting the bone-implant contact (A), bone area (B), and bone area inside the perforating hole $(\mathbf{C})$ at 3 weeks and I 2 weeks of healing. Note: *Represents a statistical significance with a $P$-value $<0.05$.

Abbreviations: BIC, bone-implant contact; BA, bone area.
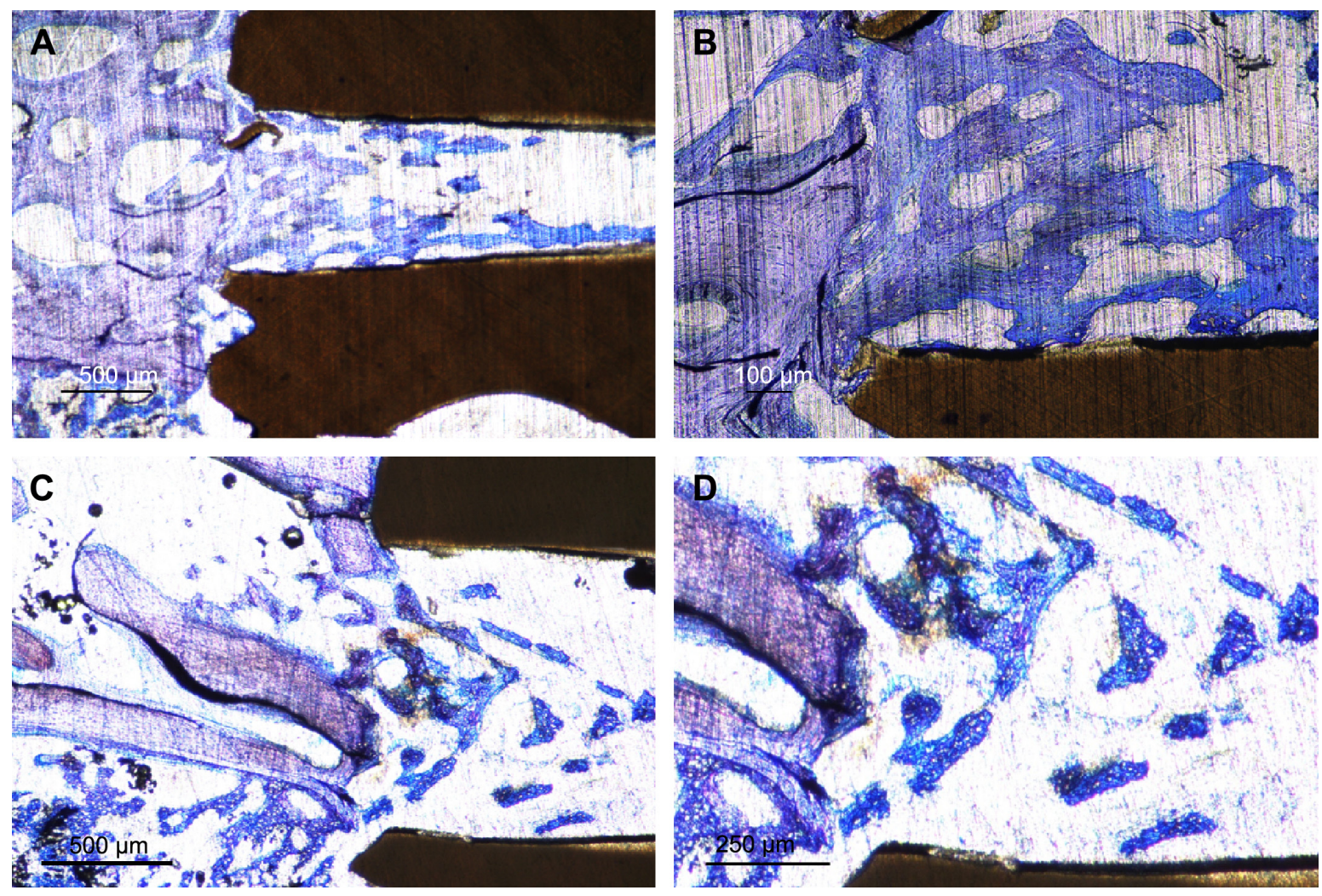

Figure 7 Histological sections in two magnifications showing the apical perforation of HA-coated (A and $\mathbf{B})$ and uncoated (C and $\mathbf{D})$ PEEK implants, 3 weeks after insertion.

Notes: The HA-coated implant in lower magnification $(\mathbf{A})$ shows bone growth through the entire hole. In the enlarged image (B), the line between the new bone and old bone can be tracked, and inside the hole, the bone approaches the implant surface with regular intervals through the hole. The uncoated PEEK implant demonstrates significantly less bone area at the entrance and inside the perforated hole compared to that of HA-coated PEEK (C). With the higher magnification, the bone structure was found to be located in the center of the perforation without having contact to the implant surface (D).

Abbreviations: HA, hydroxyapatite; PEEK, polyether ether ketone. 
healing times. However, the BA in the threads did not show any differences between the two groups at any healing point. Moreover, the perforating hole in the apical part of the implant revealed a clear and significant increased BA for the HAcoated PEEK implants. There are several possible explanations for these findings. One major difference was the direct contact with native bone for the threads after insertion, while the hole was only filled with blood without any initial bone contact. The regenerative process around mature bone next to the threads is different from the process inside the hole, which may affect the coating abilities. Furthermore, some of the coating may have been scraped off during insertion, despite failure to observe this in a previously conducted in vitro investigation. ${ }^{24}$ These results are consistent with the findings from several other studies that evaluated HA coating on various implant materials. ${ }^{8,25}$ Sandén et al evaluated a traditional HA coating on stainless steel in a goat model and found improved bone formation compared with untreated PEEK after 6 and 12 weeks of healing. ${ }^{26}$ However, their coating was deposited with a plasma spraying technique that provides a thicker coating without nanostructures. Similar results were published by Gu et al who demonstrated greater bone fusion for HA-coated intervertebral cages. ${ }^{27}$ Suska et al compared HA-coated PEEK and titanium with uncoated PEEK in a rabbit model and demonstrated that HA coating significantly enhances the bone response. Moreover, in the cortical bone (tibia) model, HA-coated PEEK showed significantly higher bone values than those found with HA-coated titanium implants. ${ }^{28}$ Nakahara et al revealed improved BIC for HA-coated PEEK after 6 weeks and 12 weeks of unloaded healing. The authors indicate a correlation between BIC and shear strength, both of which were enhanced for HA-coated implants compared to noncoated implants. ${ }^{29}$ Our research team found a similar correlation as Nakahara et al with our previous study using an identical implant design. The increased removal torque for the HA-coated implants compared to uncoated controls is correlated to the higher BIC for test implants. ${ }^{23}$

Another previous study by our team evaluated the same implant design and surface modification inserted in the rabbit tibia. The results revealed a significantly higher BIC and BA after 3 weeks for HA-coated implants. ${ }^{24}$ However, after 12 weeks, the BIC in the 12-week group did not show any differences between the groups, but the mean BA values were still significantly higher on the HA-coated implants. The gathered results from the previous and the present studies indicate a pronounced effect of the HA at the early stages, even for the part of the implant inside the bone marrow where less BIC would be expected. In the femur, on the other hand, the entire implant surface was in contact with the bone resulting in a higher percent of BIC and BA compared to the tibia. The higher BIC in the femur may be due to its higher ductile properties compared to those of tibia, which minimize compression and stress around the implant. The HA-coated implants in tibia and femur show at the early stage ( 3 weeks) a clear advantage on the BIC, but after the longer evaluation time (12 weeks), the percent of contact has almost ceased in progression when compared to uncoated implants. However, the amount of BA in the thread for both tibia and femur has almost been doubled from 3 to 12 weeks and still shows significance for the HA-coated implants.

PEEK is today used in several orthopedic therapies, including spinal fusion where the implant replaces the disc and allows a fusion of bone between the vertebras. Therefore, in this study, the implant was designed with a hole, perforating the apical part of the implant that allows evaluation of bone fusion and the coated surface's ability to induce osseointegration. As far as we know, no studies have previously been published with similar implant design and experimental model. However, some studies were performed with PEEK in different spine models of animals or humans. Pelletier et al compared uncoated PEEK and titanium in a sheep spine model and found that the bone fusion was similar for both groups. The authors concluded that PEEK may be a suitable replacement for titanium in spinal fusion procedures. ${ }^{30}$ Another study by Sinclair et al evaluated uncoated PEEK and porous tantalum in a cervical interbody fusion model in goat and found higher rate of fusion for tantalum. ${ }^{31}$

\section{Conclusion}

This study demonstrated that nano-sized HA coating on PEEK implants in rabbit femur significantly improved the BIC and demonstrated strong osseoconductive properties inside the perforated hole. In a clinical situation, this material composition may improve the outcomes of different spinal procedures where a rapid bone fusion is essential.

\section{Acknowledgment}

This study was supported by grants achieved from the Swedish Knowledge Foundation and King Gustaf V's and Queen Victoria's Freemason Foundation.

\section{Disclosure}

The authors report no conflicts of interest in this work.

\section{References}

1. Park P, Garton HJ, Gala VC, Hoff JT, McGillicuddy JE. Adjacent segment disease after lumbar or lumbosacral fusion: review of the literature. Spine. 2004;29(17):1938-1944. 
2. Anandjiwala J, Seo JY, Ha KY, Oh IS, Shin DC. Adjacent segment degeneration after instrumented posterolateral lumbar fusion: a prospective cohort study with a minimum five-year follow-up. Eur Spine J. 2011;20(11):1951-1960.

3. Ha SW, Kirch M, Birchler F, et al. Surface activation of polyetheretherketone (PEEK) and formation of calcium phosphate coatings by precipitation. J Mater Sci Mater Med. 1997;8(11):683-690.

4. Kurtz SM, Devine JN. PEEK biomaterials in trauma, orthopedic, and spinal implants. Biomaterials. 2007;28(32):4845-4869.

5. Chou WK, Chien A, Wang JL. Biomechanical analysis between PEEK and titanium screw-rods spinal construct subjected to fatigue loading. J Spinal Disord Tech. 2015;28(3):E121-E125.

6. Steinberg EL, Rath E, Shlaifer A, Chechik O, Maman E, Salai M. Carbon fiber reinforced PEEK Optima - a composite material biomechanical properties and wear/debris characteristics of CF-PEEK composites for orthopedic trauma implants. J Mech Behav Biomed Mater. 2013; 17:221-228.

7. Mohammadi S, Esposito M, Hall J, Emanuelsson L, Krozer A, Thomsen P. Short-term bone response to titanium implants coated with thin radiofrequent magnetron-sputtered hydroxyapatite in rabbits. Clin Implant Dent Relat Res. 2003;5(4):241-253.

8. Barkarmo S, Andersson M, Currie F, et al. Enhanced bone healing around nanohydroxyapatite-coated polyetheretherketone implants: an experimental study in rabbit bone. J Biomater Appl. 2014;29(5):737-747.

9. Katzer A, Marquardt H, Westendorf J, Wening JV, von Foerster G. Polyetheretherketone - cytotoxicity and mutagenicity in vitro. Biomaterials. 2002;23(8):1749-1759.

10. Morrison C, Macnair R, MacDonald C, Wykman A, Goldie I, Grant MH. In vitro biocompatibility testing of polymers for orthopaedic implants using cultured fibroblasts and osteoblasts. Biomaterials. 1995;16(13): 987-992.

11. Rivard $\mathrm{CH}$, Rhalmi S, Coillard C. In vivo biocompatibility testing of peek polymer for a spinal implant system: a study in rabbits. $J$ Biomed Mater Res. 2002;62(4):488-498.

12. Deligianni DD, Katsala ND, Koutsoukos PG, Missirlis YF. Effect of surface roughness of hydroxyapatite on human bone marrow cell adhesion, proliferation, differentiation and detachment strength. Biomaterials. 2001;22(1):87-96.

13. Converse GL, Yue W, Roeder RK. Processing and tensile properties of hydroxyapatite-whisker-reinforced polyetheretherketone. Biomaterials. 2007;28(6):927-935.

14. Wong KL, Wong CT, Liu WC, et al. Mechanical properties and in vitro response of strontium-containing hydroxyapatite/polyetheretherketone composites. Biomaterials. 2009;30(23-24):3810-3817.

15. Abu Bakar MS, Cheng MH, Tang SM, et al. Tensile properties, tension-tension fatigue and biological response of polyetheretherketonehydroxyapatite composites for load-bearing orthopedic implants. Biomaterials. 2003;24(13):2245-2250.

16. Cheang P, Khor KA. Addressing processing problems associated with plasma spraying of hydroxyapatite coatings. Biomaterials. 1996; 17(5):537-544.
17. Rokkum M, Reigstad A, Johansson CB. HA particles can be released from well-fixed HA-coated stems: histopathology of biopsies from 20 hips 2-8 years after implantation. Acta Orthop Scand. 2002; 73(3):298-306.

18. Albrektsson T. Hydroxyapatite-coated implants: a case against their use. J Oral Maxillofac Surg. 1998;56(11):1312-1326.

19. Meirelles L, Arvidsson A, Andersson M, Kjellin P, Albrektsson T, Wennerberg A. Nano hydroxyapatite structures influence early bone formation. J Biomed Mater Res A. 2008;87(2):299-307.

20. Svanborg LM, Hoffman M, Andersson M, Currie F, Kjellin P, Wennerberg A. The effect of hydroxyapatite nanocrystals on early bone formation surrounding dental implants. Int J Oral Maxillofac Surg. 2011;40(3):308-315.

21. Jimbo R, Xue Y, Hayashi M, et al. Genetic responses to nanostructured calcium-phosphate-coated implants. J Dent Res. 2011;90(12): 1422-1427.

22. Kjellin P, Andersson M, inventors; Promimic Ab, assignee. Synthetic nano-sized crystalline calcium phosphate and method of production. United States patent US 8206813 B2. 2012 Jun 26.

23. Johansson P. Biomechanical evaluation and surface characterization of a nano modified surface on PEEK implants: a study in the rabbit tibia. Int J Nanomedicine. 2014;9:3903-3911.

24. Johansson P, Jimbo R, Kozai Y, et al. Nanosized hydroxyapatite coating on PEEK implants enhances early bone formation: a histological and three-dimensional investigation in rabbit bone. Materials. 2015;8(7):3815.

25. Pang KM, Lee JK, Seo YK, Kim SM, Kim MJ, Lee JH. Biologic properties of nano-hydroxyapatite: an in vivo study of calvarial defects, ectopic bone formation and bone implantation. Biomed Mater Eng. 2015;25(1):25-38.

26. Sandén B, Olerud C, Johansson C, Larsson S. Improved bone-screw interface with hydroxyapatite coating: an in vivo study of loaded pedicle screws in sheep. Spine. 2001;26(24):2673-2678.

27. Gu Y, Zhang F, Lineaweaver WC, et al. In vivo study of hydroxyapatitecoated hat type cervical intervertebral fusion cage combined with IGF-I and TGF-beta1 in the goat model. J Spinal Disord Tech. Epub 2013 Nov 8.

28. Suska F, Omar O, Emanuelsson L, et al. Enhancement of CRF-PEEK osseointegration by plasma-sprayed hydroxyapatite: a rabbit model. J Biomater Appl. 2014;29(2):234-242.

29. Nakahara I, Takao M, Goto T, Ohtsuki C, Hibino S, Sugano N. Interfacial shear strength of bioactive-coated carbon fiber reinforced polyetheretherketone after in vivo implantation. J Orthop Res. 2012; 30(10):1618-1625.

30. Pelletier M, Cordaro N, Lau A, Walsh WR. PEEK versus Ti Interbody fusion devices: resultant fusion, bone apposition, initial and 26 week biomechanics. J Spinal Disord Tech. Epub 2012 Jul 13.

31. Sinclair SK, Konz GJ, Dawson JM, Epperson RT, Bloebaum RD. Host bone response to polyetheretherketone versus porous tantalum implants for cervical spinal fusion in a goat model. Spine. 2012;37(10): E571-E580
International Journal of Nanomedicine

\section{Publish your work in this journal}

The International Journal of Nanomedicine is an international, peerreviewed journal focusing on the application of nanotechnology in diagnostics, therapeutics, and drug delivery systems throughout the biomedical field. This journal is indexed on PubMed Central, MedLine, CAS, SciSearch ${ }^{\circledR}$, Current Contents ${ }^{\circledR} /$ Clinical Medicine,

\section{Dovepress}

Journal Citation Reports/Science Edition, EMBase, Scopus and the Elsevier Bibliographic databases. The manuscript management system is completely online and includes a very quick and fair peer-review system, which is all easy to use. Visit http://www.dovepress.com/ testimonials.php to read real quotes from published authors. 